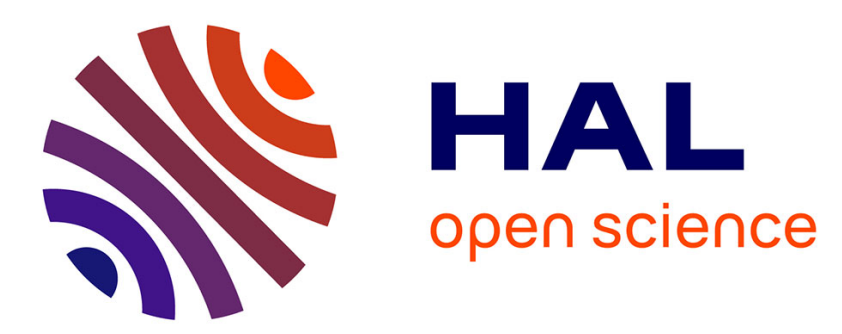

\title{
Propagation multi-modale dans les systèmes périodiques couplés
}

\author{
Jean-Mathieu Mencik, Mohamed Ichchou, Louis Jézéquel
}

\section{To cite this version:}

Jean-Mathieu Mencik, Mohamed Ichchou, Louis Jézéquel. Propagation multi-modale dans les systèmes périodiques couplés. 7e colloque national en calcul des structures, CSMA, May 2005, Giens, France. hal-01813048

\author{
HAL Id: hal-01813048 \\ https://hal.science/hal-01813048
}

Submitted on 12 Jun 2018

HAL is a multi-disciplinary open access archive for the deposit and dissemination of scientific research documents, whether they are published or not. The documents may come from teaching and research institutions in France or abroad, or from public or private research centers.
L'archive ouverte pluridisciplinaire HAL, est destinée au dépôt et à la diffusion de documents scientifiques de niveau recherche, publiés ou non, émanant des établissements d'enseignement et de recherche français ou étrangers, des laboratoires publics ou privés. 


\title{
Propagation multi-modale dans les systèmes périodiques couplés
}

\author{
Jean-Mathieu Mencik — Mohamed Ichchou — Louis Jézéquel \\ Laboratoire de Tribologie et Dynamique des Systèmes \\ Ecole Centrale de Lyon \\ 36 Avenue Guy de Collongue \\ Bâtiment E6 \\ 69134 ECULLY Cedex \\ Jean-Mathieu.Mencik@ec-lyon.fr
}

RÉSUMÉ. Cette étude propose une description numérique du comportement vibratoire large bande d'un ensemble de systèmes complexes périodiques - chaque système est composé, selon une direction spécifique, de sous-structures identiques - élastiques et dissipatifs, couplés. Les variables cinématiques (déplacements, forces) de chaque système sont décrites sur la base des modes d'ondes se propageant selon la direction de périodicité. L'évolution fréquentielle de chaque mode est analysée (mise en évidence des courbes de dispersion). Les propriétés de diffusion (réflection et transmission) des modes de différents systèmes périodiques couplés par un élément élastique sont formulées.

ABSTRACT. The present work is concerned with a numerical description of the wide band vibratory behavior of a set of coupled elastic and dissipative complex periodic systems - each system is composed, along a specific direction, of identical substructures. The kinematic quantities (displacements, forces) of each system are described from the wave modes propagating along the direction of periodicity. The frequency evolution of each mode is analyzed (evaluation of the dispersion curves). The diffusion properties (reflection and transmission) of the modes of different systems coupled through an elastic element are formulated.

MOTS-CLÉS: Système périodique, sous-structure, mode d'onde, guide d'ondes, modèle éléments finis, courbe de dispersion, élément de couplage, multiplicateur de Lagrange, matrice de diffusion.

KEYWORDS: Periodic system, substructure, wave mode, waveguide, finite element model, dispersion curve, coupling element, Lagrange multiplier, diffusion matrix. 


\section{Introduction}

Cette étude s'inscrit dans l'analyse du comportement vibratoire large bande (spécifiquement en MF et HF) d'un ensemble de systèmes solides, élancés (poutres, ... .), couplés. On suppose que chaque système est élastique, dissipatif et périodique - c'està-dire composé, selon une direction donnée, de sous-structures identiques pouvant être complexes [ZHO 95]. Dans l'optique où l'on désire s'affranchir d'une description numérique «classique »(3D) de ces systèmes (par la Méthode des Elements Finis), coûteuse dès lors que l'on s'intéresse aux domaines MF et HF (nécessité d'un maillage fin de chaque système adapté aux faibles longueurs d'ondes), on adopte une description 1D simplifiée (hypothèse des guides d'ondes) : en accord avec le théorème de Bloch, les variables cinématiques (déplacements, forces) de chaque système sont décrites sur la base des modes d'ondes se propageant selon la direction de périodicité (approche multi-modale). Pour un système donné, ces modes sont obtenus numériquement à partir du modèle éléments finis d'une sous-structure donnée. Après un rappel sur la notion de propagation dans un solide élastique et périodique, on propose une description de la matrice de diffusion (coefficients de réflection et de transmission) des modes de plusieurs guides d'ondes couplés via un élément élastique.

\section{Propagation dans un guide d'onde périodique}

On s'intéresse au comportement dynamique d'une structure élancée, telle que décrite sur la Figure 1, composée, selon une direction spécifique (axe $x$ ), de $N$ sousstructures identiques, élastiques, linéaires et dissipatives. On note que cette description générale s'applique aux systèmes à sections constantes. Sous l'hypothèse de guide d'ondes [BRI 46], on montre que la dynamique du système global se formule sur la base des ondes se propageant selon l'axe $x$.

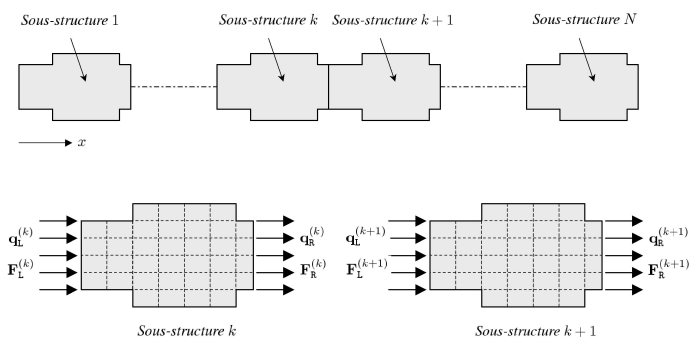

Figure 1. Illustration d'un guide d'ondes périodique.

Considérons le modèle élément finis d'une sous-structure $k$ donnée $(k=1, \ldots, N)$ appartenant au système global. Les frontières gauche et droite de la sous-structure discrétisée sont supposées contenir $n$ ddl's. Les variables cinématiques - déplacements $\mathbf{q}$ et forces $\mathbf{F}$ - définies sur ces frontières sont notées $\left(\mathbf{q}_{\mathrm{L}}, \mathbf{q}_{\mathrm{R}}\right)$ et $\left(\mathbf{F}_{\mathrm{L}}, \mathbf{F}_{\mathrm{R}}\right)$, respectivement (cf. Figure 1), et sont représentées à partir des vecteurs d'états $\mathbf{u}_{\mathrm{L}}^{(k)}=$ 
$\left(\left(\mathbf{q}_{\mathrm{L}}^{(k)}\right)^{\mathrm{T}}\left(-\mathbf{F}_{\mathrm{L}}^{(k)}\right)^{\mathrm{T}}\right)^{\mathrm{T}}$ et $\mathbf{u}_{\mathrm{R}}^{(k)}=\left(\left(\mathbf{q}_{\mathrm{R}}^{(k)}\right)^{\mathrm{T}}\left(\mathbf{F}_{\mathrm{R}}^{(k)}\right)^{\mathrm{T}}\right)^{\mathrm{T}}$. En exprimant les relations de couplage à la jonction de deux sous-structures consécutives $k$ et $k-1(k=2, \ldots, N)$ $\mathbf{u}_{\mathrm{L}}^{(k)}=\mathbf{u}_{\mathrm{R}}^{(k-1)}$ - les vecteurs d'états $\mathbf{u}_{\mathrm{L}}^{(k)}$ et $\mathbf{u}_{\mathrm{L}}^{(k-1)}$ sont reliés de la façon suivante :

$$
\mathbf{u}_{\mathrm{L}}^{(k)}=\mathbf{S u}_{\mathrm{L}}^{(k-1)} \quad \forall k \in\{2, \ldots, N\}
$$

où $\mathbf{S}$ est une matrice symplectique [ZHO 95]. En accord avec le théorème de Bloch [BRI 46], les solutions $\mathbf{u}_{\mathrm{L}}$ sont de la forme $\mathbf{u}_{\mathrm{L}}^{(k)}=\mu \mathbf{u}_{\mathrm{L}}^{(k-1)}$ et dérivent d'un problème aux valeurs propres :

$$
\mathbf{S} \boldsymbol{\Phi}_{i}=\mu_{i} \boldsymbol{\Phi}_{i} \quad, \quad\left|\mathbf{S}-\mu_{i} \mathbf{I}_{2 n}\right|=\mathbf{0} .
$$

Les solutions $\left\{\left(\mu_{i}, \boldsymbol{\Phi}_{i}\right)\right\}_{i=1, \ldots, 2 n}$ définissent les modes d'ondes du système périodique. La matrice $\boldsymbol{\Phi}$ des vecteurs propres peut s'écrire

$$
\boldsymbol{\Phi}=\left[\begin{array}{ll}
\boldsymbol{\Phi}_{\mathbf{q}}^{\text {inc }} & \boldsymbol{\Phi}_{\mathbf{q}}^{\mathrm{ref}} \\
\boldsymbol{\Phi}_{\mathbf{F}}^{\text {inc }} & \boldsymbol{\Phi}_{\mathbf{F}}^{\text {ref }}
\end{array}\right]
$$

où $\boldsymbol{\Phi}^{\text {inc }}=\left(\left(\boldsymbol{\Phi}_{\mathbf{q}}^{\text {inc }}\right)^{\mathrm{T}}\left(\boldsymbol{\Phi}_{\mathbf{F}}^{\text {inc }}\right)^{\mathrm{T}}\right)^{\mathrm{T}}$ et $\boldsymbol{\Phi}^{\text {ref }}=\left(\left(\boldsymbol{\Phi}_{\mathbf{q}}^{\text {ref }}\right)^{\mathrm{T}}\left(\boldsymbol{\Phi}_{\mathbf{F}}^{\text {ref }}\right)^{\mathrm{T}}\right)^{\mathrm{T}}$ sont des matrices de taille identique $(2 n \times n)$ et représentent les modes incidents et réfléchis par une limite du guide [ZHO 95, YON 89]. Le suivi de chaque mode dans le domaine fréquentiel est respecté en observant les propriétés d'orthogonalité symplectique des vecteurs propres $\left\{\boldsymbol{\Phi}_{i}\right\}_{i}$ [ZHO 95] : si $\boldsymbol{\Phi}_{i}(\omega)$ représente le $i$ ème mode à la fréquence $\omega$, alors le $i$ ème mode $\boldsymbol{\Phi}_{i}\left(\omega+\Delta_{\omega}\right)$ à la fréquence $\omega+\Delta_{\omega}$ doit être choisi de telle manière que la quantité

$$
A_{i}(\omega)=\left(\boldsymbol{\Phi}_{l}(\omega)\right)^{\mathrm{T}} \mathbf{J}_{n} \boldsymbol{\Phi}_{i}\left(\omega+\Delta_{\omega}\right) \quad, \quad \mu_{l}(\omega)=1 / \mu_{i}(\omega)
$$

est maximale. La validité de ce critère est basée sur l'hypothèse que le pas fréquentiel $\Delta_{\omega}$ est suffisamment petit de sorte que le vecteur propre $\boldsymbol{\Phi}_{i}$ varie faiblement entre $\omega$ et $\omega+\Delta_{\omega}$. Finalement, les vecteurs d'états $\mathbf{u}_{\mathrm{L}}^{(k)}$ et $\mathbf{u}_{\mathrm{R}}^{(k)}$ d'une sous-structure $k$ donnée peuvent s'exprimer à partir de la matrice des vecteurs propres $\Phi$ [ZHO 95] :

$$
\mathbf{u}^{(k)}=\mathbf{\Phi} \mathbf{Q}^{(k)} \quad \forall k \in\{1, \ldots, N\} \quad, \quad \mathbf{Q}=\left(\begin{array}{c}
\mathbf{Q}^{\text {inc }} \\
\mathbf{Q}^{\text {ref }}
\end{array}\right)
$$

où $\mathbf{Q}$ désignent les amplitudes des modes d'ondes, pouvant être décomposées en partie incidente $\mathbf{Q}^{\text {inc }}$ et réfléchie $\mathbf{Q}^{\text {ref }}$.

\section{Relations de couplage entre guides d'ondes}

On formule les coefficients de diffusion (réflection et transmission) des modes d'ondes de plusieurs systèmes couplés. Cette étude est basée sur l'analyse de deux guides d'ondes couplés. D'une manière générale, les deux systèmes sont supposés être connectés via un élément de couplage élastique (voir Figure 2). 


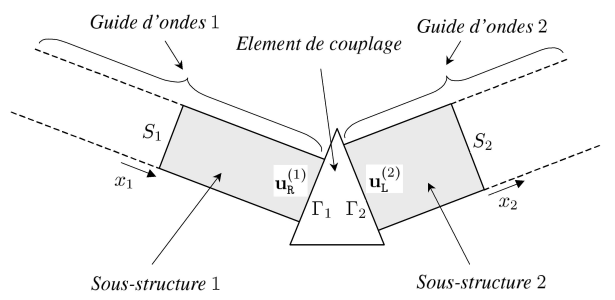

Figure 2. Illustration de deux guides d'ondes couplés.

On considère deux sous-structures (1 et 2), telles que définies dans la section 2 , situées aux extrémités des guides d'ondes (cf. Figure 2). Ces sous-structures sont couplés avec l'élément de couplage sur les surfaces $\Gamma_{1}$ et $\Gamma_{2}$ et sont couplés avec les autres sous-structures, à l'intérieur des guides d'ondes, sur les surfaces $S_{1}$ et $S_{2}$. On suppose qu'il n'y a pas de source d'excitation à l'intérieur de l'élément de couplage. La continuité du déplacement sur $\Gamma_{1}$ et $\Gamma_{2}$ est imposée en introduisant deux champs de multiplicateurs de Lagrange $\boldsymbol{\lambda}_{1}$ et $\boldsymbol{\lambda}_{2}$ [OHA 97]. la formulation variationnelle pour les deux sous-structures et l'élément de couplage s'écrit :

$$
\begin{aligned}
& \sum_{i=1,2}\left(-\omega^{2} \int_{\Omega_{i}} \rho\left(\delta \mathbf{w}^{(i)}\right)^{\mathrm{T}} \mathbf{w}^{(i)} d \mathbf{x}+\int_{\Omega_{i}}\left(\boldsymbol{\epsilon}\left(\delta \mathbf{w}^{(i)}\right)\right)^{\mathrm{T}} \boldsymbol{\sigma}\left(\mathbf{w}^{(i)}\right) d \mathbf{x}\right) \\
& +\left(-\omega^{2} \int_{\Omega_{\mathrm{c}}} \rho\left(\delta \mathbf{w}^{\mathrm{c}}\right)^{\mathrm{T}} \mathbf{w}^{\mathrm{c}} d \mathbf{x}+\int_{\Omega_{\mathrm{c}}}\left(\boldsymbol{\epsilon}\left(\delta \mathbf{w}^{\mathrm{c}}\right)\right)^{\mathrm{T}} \boldsymbol{\sigma}\left(\mathbf{w}^{\mathrm{c}}\right) d \mathbf{x}\right) \\
& +\int_{\Gamma_{1}}\left(\delta \boldsymbol{\lambda}_{1}\right)^{\mathrm{T}}\left(\mathbf{w}^{\mathrm{c}}-\mathbf{w}^{(1)}\right) d s(\mathbf{x})+\int_{\Gamma_{2}}\left(\delta \boldsymbol{\lambda}_{2}\right)^{\mathrm{T}}\left(\mathbf{w}^{\mathrm{c}}-\mathbf{w}^{(2)}\right) d s(\mathbf{x}) \\
& +\int_{\Gamma_{1}}\left(\delta \mathbf{w}^{\mathrm{c}}-\delta \mathbf{w}^{(1)}\right) \boldsymbol{\lambda}_{1} d s(\mathbf{x})+\int_{\Gamma_{2}}\left(\delta \mathbf{w}^{\mathrm{c}}-\delta \mathbf{w}^{(2)}\right) \boldsymbol{\lambda}_{2} d s(\mathbf{x}) \\
& =\int_{S_{1}}\left(\delta \mathbf{w}^{(1)}\right)^{\mathrm{T}} \mathbf{f}^{(1)} d s(\mathbf{x})+\int_{S_{2}}\left(\delta \mathbf{w}^{(2)}\right)^{\mathrm{T}} \mathbf{f}^{(2)} d s(\mathbf{x}),
\end{aligned}
$$

où $\mathbf{w}^{(i)}$ et $\mathbf{w}^{\mathrm{c}}$ représentent les champs de déplacements de la sous-structure $i(i=$ $1,2)$ et de l'élément de couplage, respectivement; $\mathbf{f}^{(i)}(i=1,2)$ représente la force appliquée sur $S_{i}$. Dans le cadre de la Méthode des Elements Finis, les champs de déplacements et de multiplicateurs de Lagrange sont discrétisés. L'équilibre dynamique des sous-structures couplés avec l'élément de couplage permet alors d'exprimer une relation entre les forces $\left(\mathbf{F}_{\mathrm{R}}^{(1)}, \mathbf{F}_{\mathrm{L}}^{(2)}\right)$ appliqués sur les ddl's des frontières droite et gauche des sous-structures 1 et 2 et les déplacements $\left(\mathbf{q}_{R}^{(1)}, \mathbf{q}_{\mathbf{L}}^{(2)}\right)$ correspondants :

$$
-\mathbf{T}^{\mathbf{T}} \mathbb{K}^{*} \mathbf{T}\left(\begin{array}{c}
\mathbf{q}_{\mathrm{R}}^{(1)} \\
\mathbf{q}_{\mathrm{L}}^{(2)}
\end{array}\right)=\left(\begin{array}{c}
\mathbf{F}_{\mathrm{R}}^{(1)} \\
\mathbf{F}_{\mathrm{L}}^{(2)}
\end{array}\right) .
$$


La matrice $\mathbb{K}^{*}$ représente l'opérateur de rigidité dynamique de l'élément de couplage qui est symétrique; la matrice $\mathbf{T}$ traduit l'influence des multiplicateurs de Lagrange. En considérant (5), l'équation (7) permet de relier, à partir d'une matrice de diffusion $\mathbb{C}$, les amplitudes $\left(\mathbf{Q}^{\text {ref }(1)}, \mathbf{Q}^{\text {ref(2)}}\right)$ des modes (guides d'ondes 1 et 2 ) réfléchies par l'élément de couplage et les amplitudes $\left(\mathbf{Q}^{\text {inc(1) }}, \mathbf{Q}^{\text {inc(2) }}\right)$ des modes incidents :

$$
\left(\begin{array}{l}
\mathbf{Q}^{\operatorname{ref}(1)} \\
\mathbf{Q}^{\operatorname{ref}(2)}
\end{array}\right)=\mathbb{C}\left(\begin{array}{l}
\mathbf{Q}^{\mathrm{inc}(1)} \\
\mathbf{Q}^{\operatorname{inc}(2)}
\end{array}\right)
$$

La matrice de diffusion $\mathbb{C}$ s'exprime :

$$
\mathbb{C}=-\left[\mathbf{T}^{\mathrm{T}} \mathbb{K}^{*} \mathbf{T} \boldsymbol{\Psi}_{\mathrm{q}}^{\mathrm{ref}}+\mathbf{\Psi}_{\mathbf{F}}^{\mathrm{ref}}\right]^{-1}\left[\mathbf{T}^{\mathrm{T}} \mathbb{K}^{*} \mathbf{T} \boldsymbol{\Psi}_{\mathrm{q}}^{\text {inc }}+\boldsymbol{\Psi}_{\mathbf{F}}^{\text {inc }}\right]
$$

où

$$
\begin{aligned}
& \boldsymbol{\Psi}_{\mathbf{q}}^{\text {inc }}=\left[\begin{array}{cc}
\boldsymbol{\Phi}_{\mathbf{q}}^{\text {inc }(1)} & \mathbf{0} \\
\mathbf{0} & \boldsymbol{\Phi}_{\mathbf{q}}^{\text {inc }(2)}
\end{array}\right] \quad, \quad \Psi_{\mathbf{q}}^{\mathrm{ref}}=\left[\begin{array}{cc}
\boldsymbol{\Phi}_{\mathbf{q}}^{\mathrm{ref}(1)} & \mathbf{0} \\
\mathbf{0} & \boldsymbol{\Phi}_{\mathbf{q}}^{\mathrm{ref}(2)}
\end{array}\right] \\
& \boldsymbol{\Psi}_{\mathbf{F}}^{\text {inc }}=\left[\begin{array}{cc}
\boldsymbol{\Phi}_{\mathbf{F}}^{\text {inc }(1)} & \mathbf{0} \\
\mathbf{0} & \boldsymbol{\Phi}_{\mathbf{F}}^{\text {inc(2) }}
\end{array}\right] \quad, \quad \boldsymbol{\Psi}_{\mathbf{F}}^{\mathrm{ref}}=\left[\begin{array}{cc}
\boldsymbol{\Phi}_{\mathbf{F}}^{\mathrm{ref}(1)} & \mathbf{0} \\
\mathbf{0} & \boldsymbol{\Phi}_{\mathbf{F}}^{\mathrm{ref}(2)}
\end{array}\right] .
\end{aligned}
$$

Une extension de cette étude à l' analyse de $M$ guides d'ondes couplés ne pose pas de problème en considérant un raisonnement similaire à celui exposé dans cette section.

\section{Résultats numériques}

On considère deux guides d'ondes homogènes de sections rectangulaires couplés selon la direction transversale (axe $z$ ) par un ressort linéaire de raideur $K^{\prime}=$ $10^{9} \mathrm{~N} / \mathrm{m}$ (cf. Figure 3). Les caractéristiques du guide d'ondes 1 sont : densité $\rho^{(1)}=$ $7800 \mathrm{~kg} / \mathrm{m}^{3}$, module d'Young $E^{(1)}=2 \times 10^{11} \mathrm{~Pa}$, amortissement $\eta^{(1)}=10^{-2}$, section $h_{y}^{(1)} \times h_{z}^{(1)}$, où $h_{y}^{(1)}=30 \times 10^{-2} m$ et $h_{z}^{(1)}=20 \times 10^{-2} \mathrm{~m}$. Les caractéristiques du guide d'ondes 2 sont : densité $\rho^{(2)}=2700 \mathrm{~kg} / \mathrm{m}^{3}$, module d'Young $E^{(2)}=$ $7 \times 10^{10} P a$, amortissement $\eta^{(2)}=10^{-2}$, section $h_{y}^{(2)} \times h_{z}^{(2)}$, où $h_{y}^{(2)}=24 \times 10^{-2} m$ et $h_{z}^{(2)}=20 \times 10^{-2} \mathrm{~m}$. Le modèle éléments finis d'une sous-structure (cf. section 2) est illustré sur la Figure 3.

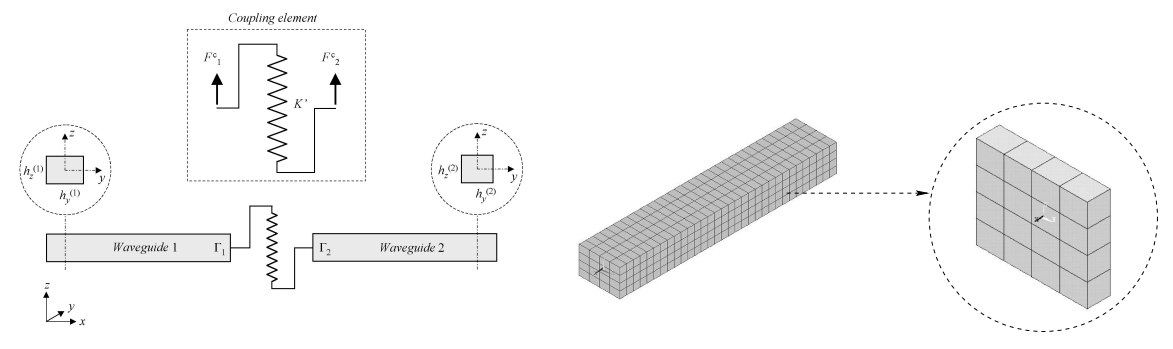

Figure 3. Deux guides d'ondes homogènes couplés transversalement par un ressort linéaire et modèle éléments finis d'une sous-structure. 
Sur la Figure 4, on a représenté dans la bande de fréquences [ $40 \mathrm{~Hz}, 8000 \mathrm{~Hz}$ ] les courbes de dispersion pour le guide d'ondes 1 des modes propagatifs et qui deviennent propagatifs (modes de section). On a représenté les coefficients de diffusion (réflection et transmission) des modes de flexion des deux guides d'ondes. Les résultats obtenus par l'approche multi-modale sont clairement validé en BF comparativement à la théorie de l'élasticité linéaire. A mesure que la fréquence augmente, les deux solutions diffèrent. Ces différences s'expliquent de la manière suivante : (i) la description en ondes planes n'est plus valide en MF et HF; (ii) dans l'approche multi-modale, les modes de flexion échangent de l'énergie avec les modes de section.
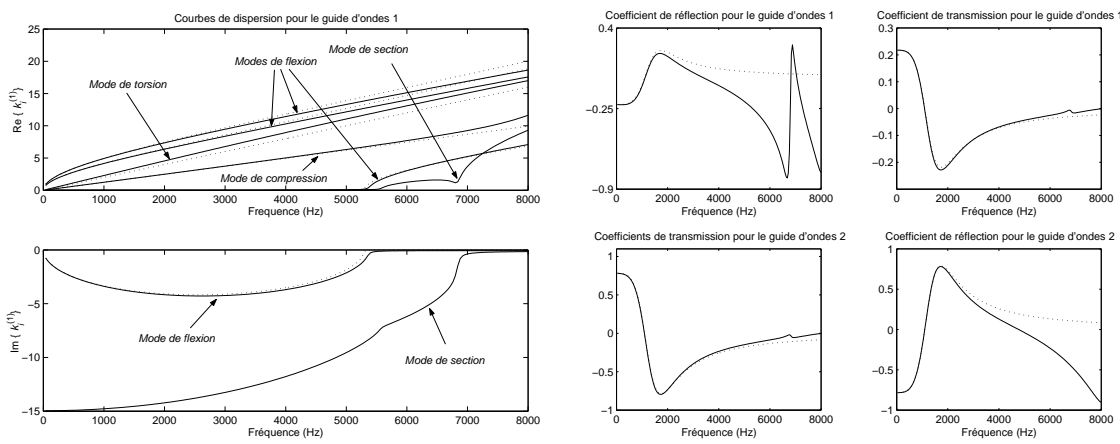

Figure 4. Courbes de dispersion et coefficients de diffusion (parties réelles) obtenus par l'approche multi-modale (-) et par la théorie de l'élasticité linéaire (…).

\section{Conclusion}

On a modélisé la diffusion des ondes de plusieurs systèmes périodiques couplés via un élément élastique. Le modèle proposé est valide en MF et HF puisqu'il prend en compte les modes de section apparaissant aux fréquences élevées. L'approche multimodale a été validée en BF dans le cas de deux systèmes homogènes couplés.

\section{Bibliographie}

[BRI 46] BRILlouin L., Wave Propagation in Periodic Structures, Mc Graw Hill Publishing Compagny, New York, 1946.

[OHA 97] OHAYON R., SAmpaio R., SoIZE C., « Dynamic substructuring of damped structures using singular value decomposition », Transactions of the ASME, vol. 64, 1997, p. 292-298.

[YON 89] YoNG Y., LIN Y. K., « Propagation of decaying waves in periodic and piecewise periodic structures of finite length », Journal of Sound and Vibration, vol. 129, $\mathrm{n}^{\circ} 2,1989$, p. 99-118.

[ZHO 95] ZHONG W. X., Williams F. W., « On the direct solution of wave propagation for repetitive structures », Journal of Sound and Vibration, vol. 181, $\mathrm{n}^{\circ}$ 3, 1995, p. 485-501. 